\title{
El comercio en las estrategias de vertebración territorial de la Comunidad Valenciana
}

\section{Valencia 1978-2010: the management of diseconomies of agglomeration and the new city centres}

\author{
Gabino Ponce Herrero ${ }^{1}$
}

\section{INTRODUCCIÓN}

Desde 1977, la Comunidad Valenciana ha desarrollado un gran aparato legal y, en concreto, un paquete de normas específicas orientadas a la ordenación del territorio con el propósito último de «construir un nuevo país», vertebrar una región hasta entonces desmembrada y con claras tendencias centrífugas, y potenciar el rango estatal e internacional de la capital del nuevo sistema urbano «en construcción». Al mismo tiempo, se ha manejado la actividad comercial como estrategia para la configuración de espacios sociales urbanos y contrarrestar, en lo posible, las tendencias hacia la dispersión urbana.

\subsection{Marco teórico}

El nuevo modelo urbano expansivo desarrollado en las últimas décadas del siglo pasado, como estrategia que potencia su atractivo, se ha dotado de nuevos espacios comerciales periféricos, que se organizan en forma de ámbitos para el encuentro, la relación y el ocio -los centros comerciales-. Son nuevos instrumentos urbanísticos que, pese a las críticas recibidas, mantienen su fun-

\footnotetext{
${ }^{1}$ Universidad de Alicante, departamento de Geografía Humana; Gabino.ponce@ua.es; Proyecto URBSPAIN, CSO 2009-11261-Surbprograma GEOG.
} 
ción de ámbito para la cohesión social, si bien bajo fórmulas directamente subordinadas al consumo, lo que ha afectado gravemente no sólo al papel del centro urbano como crisol de relaciones sociales, sino también a la actividad comercial tanto del centro histórico como de las principales vías del Ensanche decimonónico.

El desplazamiento de esos espacios sociales hacia la periferia es estrategia manejada por los agentes que propician la ciudad expansiva, que asocian interesadamente el atractivo de un nuevo centro comercial con la promoción inmobiliaria, en un negocio complementario que genera sinergias específicas, aprovechadas tanto por los nuevos centros comerciales, como por los promotores inmobiliarios. Pero también resultado lógico de los nuevos formatos de vivienda en las periferias, en urbanizaciones dispersas y cerradas, que niegan la calle como espacio de relación y consagran toda la superficie edificable de la parcela al uso residencial, propiciando la agrupación comercial en forma de grandes centros comerciales, instalados junto a los principales nodos de comunicación.

Esas dinámicas, experimentadas en EE.UU por lo menos desde los años 1950, y en la Europa occidental al menos desde los años 1960, han eclosionado de forma subrepticia en las ciudades españolas en los años 1990, en plena crisis del control social del urbanismo, desplazado en el pensamiento general y en el marco legal (urbanístico y comercial) por la flexibilidad y permisividad postmodernas y neoliberales.

El proceso se ha dado de forma paulatina, pero rápida, conforme con el aumento de los niveles de renta y, por consiguiente de la demanda y del consumo, que han fomentado la oferta. Pero también a partir de la aceptación de la zonificación como paradigma indiscutible, desde el cual se acepta y potencia la segregación de usos en las normas urbanísticas (planes generales). La especialización de las diferentes partes de la ciudad ha fomentado el transporte privado -el automóvil- en mayor medida que el público, lo que es causa también de la centrifugación de las áreas comerciales hacia espacios periféricos muy accesibles para el automóvil.

En tales cambios puede establecerse hasta cuatro fases consecutivas, conforme con la evolución de la actividad comercial minorista, todas con grandes afectaciones en la estructura urbana y en los modos de vida:

1. Consolidación del modelo comercial liberal que gravita sobre los centros urbanos y ejerce su primacía sobre una periferia subordinada: la ciudad compacta aumenta sus densidades en los espacios centrales, de actividad comercial -y terciaria en general-y de población. 
2. Los nuevos formatos comerciales -grandes superficies (en vertical: varias plantas) y franquicias- se instalan en los principales ejes del centro urbano, potenciando su atractivo y densidad demográfica.

3. El crecimiento de los suburbios fomenta nuevas fórmulas comerciales sustentadas por la movilidad privada en automóvil. Aparecen grandes superficies (en horizontal) en los principales nodos de comunicación de la periferia, y las grandes superficies y franquicias del centro desdoblan sus equipamientos, instalando nuevas unidades en esa periferia que crece y se hace más accesible conforme con los nuevos modos de vida suburbana.

4. Se consolida la periferia suburbana con ejes y nuevas centralidades comerciales, al tiempo que el centro comercial tradicional entra en crisis. La despoblación y el envejecimiento de los barrios centrales va pareja a la degradación de la actividad comercial minorista. Mientras las nuevas tecnologías contribuyen al desarraigo territorial del comercio y favorecen el hábitat disperso.

En apenas tres décadas, en la Comunidad Valenciana se ha transitado de manera acelerada por las cuatro etapas básicas en que puede dividirse la evolución de la actividad comercial. Los cambios urbanos se han reflejado en alteraciones de los hábitos ciudadanos y, entre otros, de sus pautas de consumo, mediatizadas por el atractivo diseño de los nuevos centros comerciales, por su accesibilidad, por sus nuevas técnicas de compra y marketing y, en especial, por su vinculación con el ocio, mediante atractivas y sugerentes estrategias que explotan la vena hedonista de la población y alteran sustancialmente el modelo comercial -y de relación- de la ciudad y, con él, el mapa de los espacios para la cohesión social y sus características.

Asumidas las competencias en materia urbanística, los primeros gobiernos de la Comunidad Valenciana desarrollaron un aparato legal que pretendía regular y aprovechar el papel del comercio como herramienta de vertebración social y territorial. En ese contexto se insertan una serie de estrategias territoriales concretas -los Planes de Acción Comercial -PAC- para la salvaguarda y mantenimiento de los antiguos espacios comerciales de la ciudad (centros históricos y Ensanches), muy afectados por el cambio. Se trata de programas económicos, con un modesto soporte urbanístico, para recuperar el atractivo económico perdido y regenerar su capacidad para el encuentro y la relación.

Son medidas muy interesantes desde el punto de vista urbanístico, por cuanto, más allá de la solvencia del negocio privado, convergen en el objetivo de mantener la ciudad histórica y la ciudad compacta, servida por el trans- 
porte público y muy accesible andando, como el lugar emblemático de la cohesión social. Sin embargo, su carácter puntual -planes de acción comercial para algunas calles del centro-, si bien han contribuido al sostenimiento en el tiempo del comercio minorista tradicional, no han logrado contrarrestar los grandes movimientos centrífugos del equipamiento comercial.

\subsection{Hipótesis, objetivos y fuentes}

Para organizar el territorio valenciano como unidad sistémica, las primeras propuestas autonómicas se orientaron hacia la construcción de un modelo jerarquizado piramidal, conforme con la «Teoría de los lugares centrales» de Christaller, en el que todo el sistema se halla subordinado a la ciudad capital, que desborda su influencia por el territorio a partir de otras ciudades de diferente rango menor (COPUT, 1995). La Teoría de los lugares centrales ha tenido gran predicamento en los modelos de ciencia regional para la creación de sistemas nacionales de ciudades en la segunda mitad del siglo pasado. Y la idea de los «modelos gravitacionales» a partir de un polo de desarrollo regional ha sustentado las directrices de las políticas de cohesión de la Europa de las regiones (Comisión Europea, 1995).

Diversos estudios han analizado los resultados de esas estrategias de sesgo positivista y "moderno», que han llevado a las tierras valencianas desde una estructura fragmentada en forma de archipiélago (Salom, 1992; Ponce, 2005 y 2006; Ponce y Martí, 2011) hacia una organización piramidal que colocó en su cúspide a la ciudad de Valencia y estableció planes de carácter territorial (comarcales, subregionales y regionales) para lograr una vertebración territorial sobre la base del equilibrio jerarquizado (COPUT, 1995), entre ellos los planes de ordenación territorial del comercio analizados. No obstante, tales objetivos experimentaron un cambio de paradigma en la segunda mitad de los años 1990, con una orientación neoliberal y «postmoderna», sustentado en el pensamiento estratégico, que ha supuesto la ruptura del modelo de equilibrio territorial primigenio (Salom y Albertos, 2001; Burriel, 2008, 2009a, 2009b y 2009c; Boira, 2012; Cucó, 2013).

En ese nuevo escenario, se plantea la hipótesis de que el comercio sigue manteniendo (y ejerciendo) su potente papel como elemento vertebrador del territorio, si bien no se ponen tanto al servicio de la ciudad como al de sus propios intereses. Así, a veces convergen con las directrices urbanísticas locales, mientras que otras veces las fuerzan en beneficio propio. Se defiende que la alteración del orden lógico seguido en las políticas de planificación territo- 
rial (de abajo hacia arriba, en contra del modelo de Christaller) no han conseguido frenar la pérdida de atractivo comercial de los centros urbanos valencianos. Se plantea como objetivo fundamental analizar la eficacia de los planes de acción comercial locales frente a las dinámicas generales, haciendo hincapié en el análisis de los PAC de las dos principales ciudades. Para ello se analizan las fuentes estadísticas sobre comercio que ofrecen el INE, el DIRCE, las Cámaras de Comercio de la Comunidad Valenciana y otras fuentes, como el Anuario Económico de la Caixa y el Atlas Estadístico de las Áreas Urbanas de España.

\section{LA PLANIFICACIÓN TERRITORIAL COMO INSTRUMENTO BÁSICO}

\subsection{Planes de acción territorial y planes de acción sectorial}

La Comunidad Valenciana aprobaba en 1989 la Ley de Ordenación del Territorio (LOT) como norma fundamental para la construcción de una nueva autonomía muy afectada por tendencias centrífugas, y carente de estructuras territoriales de cohesión. La LOT repetía en la escala regional el rango de las figuras legales de planeamiento estatal anteriores: los "planes en cascada», que descendían desde el Plan Nacional (nunca desarrollado) hasta los instrumentos de planificación en la escala local, obligaban a cada plan a contemplar y respetar la figura inmediatamente superior. En la Comunidad Valenciana, el Plan Nacional o figura esencial de referencia quedaba establecido en el Avance del Plan de Desarrollo Urbanístico de la Comunidad Valenciana (COPUT, 1995), elaborado como alternativa viable a las Directrices Regionales de Ordenación del Territorio sancionadas por otras autonomías en la época (Burriel, 2009). Por debajo de él y para concretar objetivos y estrategias, se contemplaban los Planes de Acción Territorial, unos como remedo de los Planes Directores Territoriales de Coordinación de la Ley del Suelo de 1975, con el objeto de coordinar los planes generales de los diversos municipios integrados en áreas funcionales y/o comarcas, y otros, como novedad, con un singular carácter sectorial, para atender de manera integral en todo el territorio valenciano determinados aspectos considerados estratégicos, entre ellos los orientados a satisfacer de manera equilibrada y accesible una moderna provisión de bienes y servicios colectivos, en cantidad y calidad suficiente.

Se consideraron urgentes tres PAT sectoriales, que debían abordar la ordenación de tres grandes ámbitos territoriales de la Comunidad Valenciana, para la coordinación de los planes generales locales, que deberían ajustarse a las grandes 
directrices territoriales: el PAT del Litoral, el PAT del Medio Rural y el PAT de Protección de los Espacios Naturales de Interés. Sin embargo, en ese mismo año 1995 cambiaba el Gobierno regional y, con él, los paradigmas de la ordenación territorial. De manera que los PAT de carácter sectorial propuestos caerían en el olvido, cuando no en la marginación, a instancias de los intereses locales y del desarrollo del urbanismo expansivo característico de los últimos años (Burriel, 2009).

Con la nueva Ley de Ordenación del Territorio y Protección del Paisaje, en 2004 se anunciaba la elaboración de los PAT anunciados en 1995 y la redacción de otros: del paisaje, contra riesgo sísmico, de protección de la Huerta valenciana, de corredores de infraestructuras, de extracciones mineras y de recursos hídricos (Blázquez, 2007). Finalmente, tan sólo se han aprobado dos PAT sectoriales: en 2003, el que atendía a la prevención de riesgos de inundación en la Comunidad Valenciana -PATRICOVA- y en 2005 el de grandes corredores de infraestructuras.

Tal parece que la planificación territorial, pese a que el marco legal pondera sus bondades, sigue relegada, primero por la conveniencia de flexibilizar las actuaciones al margen de directrices, y luego por las urgencias desatadas en la actual crisis. De ese modo, en 2011 se aprobaba una nueva Estrategia Territorial de la Comunidad Valenciana (Decreto 1/2011, modificado por el Decreto 166/2011, del Gobierno Valenciano) con un paquete de medidas legales recogidas en las medidas Urgentes de Impulso a la Implantación de Actuaciones Territoriales Estratégicas (Decreto Ley 2/2011), para seguir insistiendo en el urbanismo a golpe de proyectos singulares, supuestamente regulados por un planeamiento territorial que no acaba de tramitarse. Más aún, si los PAT sectoriales tenían por objeto homogeneizar las actuaciones en todo el ámbito de la Comunidad Valenciana, las Actuaciones Territoriales Estratégicas proponen impulsar proyectos singulares que contribuyan a la diversificación del territorio en sus distintas escalas, «siempre que se ajusten a los principios rectores de la Estrategia Territorial de la Comunidad Valenciana» (Decreto Ley 2/2011).

\subsection{Los Planes de Acción Territorial de Comercio}

En ese contexto, en 1996 se firmaba un acuerdo entre el nuevo Gobierno Valenciano (neoliberal) y las Cámaras de Comercio de la Comunidad Valenciana, para la elaboración de un Mapa Comercial de la Comunidad Valenciana y, a partir del diagnóstico base, desarrollar un Plan de Acción Territorial para la distribución comercial de la Comunidad Valenciana -denominado PATECO- que permitiese un desarrollo armónico del sector, aportando estrategias a la inicia- 
tiva privada para la reconversión de los ámbitos afectados y para la implantación de nuevas áreas comerciales, centradas en el comercio minorista, sin embargo, como en los demás casos, no llegó a realizarse, centrándose las actuaciones en diagnósticos y propuestas de marcado carácter local.

El soporte legal lo aportaba la Ley Valenciana 8/1986, de 26 de diciembre, de Ordenación del Comercio y Superficies Comerciales, matizada por la ley estatal de 1996, de Ordenación del Comercio Minorista, que entre otras medidas planteaba varios controles y licencias para los grandes establecimientos comerciales (con al menos $2.500 \mathrm{~m}^{2}$ ) por el impacto que su apertura ocasionaba en el comercio tradicional.

La aprobación de la Directiva 2006/123/CE del Parlamento Europeo relativa al sector servicios, y la voluntad de actualizar la Ley Valenciana de 1986 llevaron a la redacción de una nueva Ley 12/2009, de 23 de diciembre, del Gobierno Valenciano en la que se modificaba la anterior en diversos aspectos, y se establecía que, en el plazo máximo de dos años, debía aprobarse el Plan de Acción Territorial Sectorial del Comercio, ajustado a los criterios contenidos en la Estrategia Territorial de la Comunidad Valenciana (de 2011). El PAT sectorial del comercio, con la denominación de PATSECOVA, debía por tanto coincidir con la Ley 3/2011, de 23 de marzo, de Comercio de la Comunidad Valenciana, sin embargo, se halla todavía en fase de exposición pública (www.patsecova.es).

\section{LOS PLANES DE ACCIÓN COMERCIAL LOCALES}

\subsection{Planes locales frente a dinámicas generales}

Sin unas claras estrategias generales para el conjunto de la Comunidad Valenciana, la transición del comercio minorista desde los centros urbanos hacia los ámbitos suburbanos se ha guiado básicamente por los intereses particulares del sector. La grave crisis comercial del centro se intentó soslayar mediante la puesta en marcha de programas locales de ayuda para la reconversión del comercio minorista tradicional hacia nuevas fórmulas más competitivas. A petición de los ayuntamientos interesados, las Cámaras de Comercio, subvencionadas por la Consellería de Economía, han elaborado diagnósticos puntuales del comercio tradicional y, a partir de ellos, han trazado estrategias para el distrito comercial afectado, a desarrollar mediante programas cofinanciados por la Generalitat y los propios ayuntamientos.

A partir de la metodología empleada en 1996 para la confección del PATECO (no aprobado), en 1997/1998 se ponían en marcha los dos primeros 
Planes de Acción Comercial -PAC- de carácter local, a petición de las ciudades de Alicante (274.577 habitantes en ese año) e Ibi (21.076 habitantes): la primera con un centro tradicional muy afectado (en 2003 se aprobaría un Plan de Recuperación Integral del Centro Tradicional), y la segunda inmersa en un interesante y exitoso proceso de reconversión industrial y económica, guiado por una dinámica agencia de desarrollo local -PROMOIBI-.

Desde entonces se han elaborado un total de 56 PAC de carácter local, tres de ámbito comarcal: los Planes Directores de Comercio de la Ribera Alta, l'Horta Nord y la Marina Alta, con el objeto de coordinar los PAC locales; y dos Estudios Territoriales (diagnósticos iniciales, sin concretar en PAC) para RequenaUtiel y para las comarcas centrales de Valencia. La crisis económica se ha dejado sentir también en la demanda de PAC, de forma que tras el impulso de 2008-2009, en el que 10 ayuntamientos vieron en estos programas una oportunidad cofinanciada de recuperación económica, en el último periodo 20122013 no se ha tramitado ninguno (figura 1).

FIGURA 1

PLANES DE ACCIÓN COMERCIAL LOCALES POR PERIODO

Comunidad Valenciana. Planes Acción Comercial

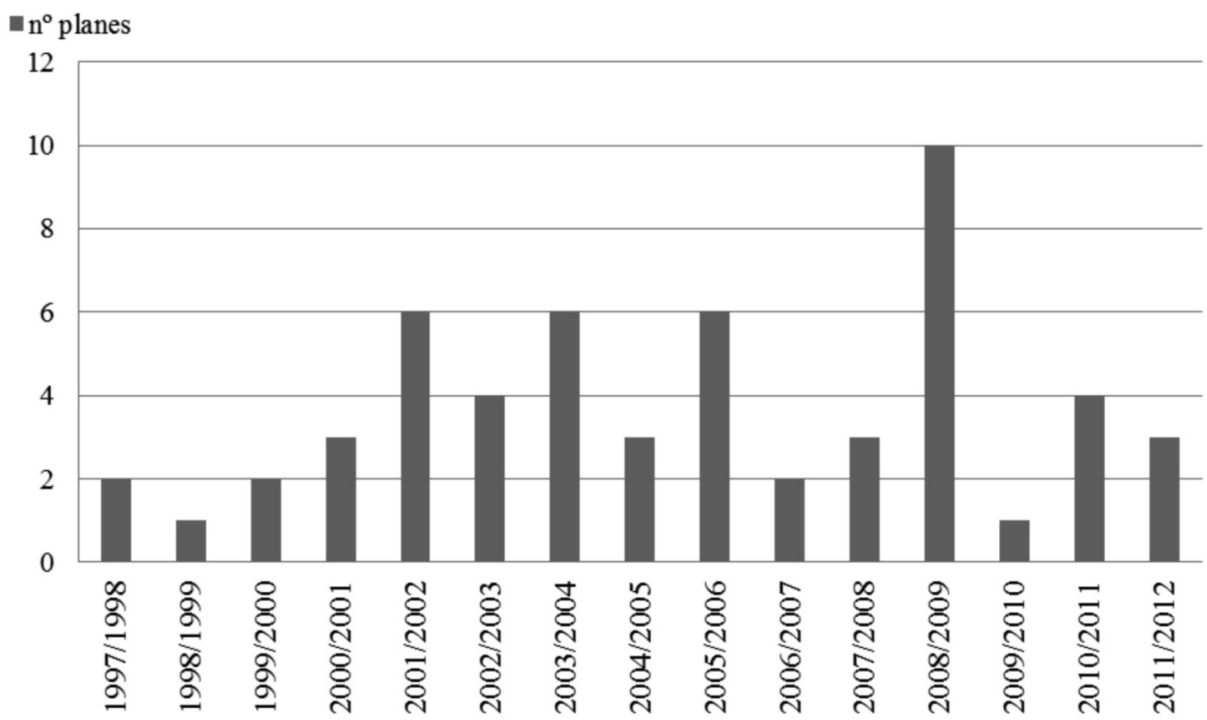

Fuente: Oficina Comercio y Territorio, 2013, elaboración propia.

Estudios Geográficos, Vol. LXXV, 276, pp. 347-372, enero-junio 2014

ISSN: 0014-1496, eISSN: 1988-8546, doi: 10.3989/estgeogr.201409 


\subsection{El mapa de los PAC}

Ya se ha comentado como la ausencia de directrices territoriales ha sido problema importante para coordinar las estrategias del comercio minorista en sus diferentes formatos (tradicional, grandes superficies, franquicias) y ámbitos (centro histórico, ensanches, periferias). También ha sido notable inconveniente para coordinar las estrategias comerciales entre municipios. Comenzar los programas desde las particularidades del barrio central de algunas ciudades, sin determinaciones vinculantes para el conjunto del municipio, no ha resuelto los problemas de centrifugado ni de deterioro. Además, a la competencia entre centro y periferia se ha sumado la desatada entre municipios vecinos. Así, pese a la bondad de los programas locales, se observa que, con frecuencia, entran claramente en conflicto y contradicciones de carácter territorial. Como en otros aspectos territoriales, también en este tema se ha invertido el orden lógico de la planificación: primero PAC locales, luego Planes Directores de Comercio de carácter comarcal (tan sólo tres: el primero en 20002001, y los otros dos en 2008-2009), mientras el verdadero PAT sectorial, el PATSECOVA, presentaba su Avance en 2012.

La propuesta de PAC ha sido pionera en el conjunto de España y exportada a otras regiones (mediante la red de Cámaras de Comercio y con fondos FEDER y del Ministerio de Economía y Competitividad) y a otros países a partir de una metodología que analiza el contexto socioeconómico del comercio minorista en la escala local y propone medidas viables (y siempre modestas) para mejorar la competitividad de los ámbitos centrales de la ciudad, sobre los que normalmente gravita la demanda de solución de problemas. Son los ayuntamientos, asociaciones de empresarios y particulares quienes deben acometer las propuestas, con el asesoramiento de la Oficina PATECO, creada a tal efecto por el Consejo de Cámaras de Comercio de la Comunidad Valenciana y que, a partir de 2009, se ha denominado Oficina de Comercio y Territorio.

Es por tanto, organismo privado, independiente del poder público, que trabaja no obstante de manera coordinada con las administraciones públicas en sus diferentes escalas, pero tiene por objetivo último favorecer el negocio privado de los empresarios - de sus asociados-. La Generalitat Valenciana financia como norma el 70\% del coste total del PAC, y el Ayuntamiento el resto (el coste medio rondaba los $40.000 €$ en los últimos planes acometidos). Si bien, desde 2008, el Gobierno valenciano ha autorizado a otras consultoras privadas la realización de otros PAC, rompiendo el monopolio de las Cámaras de Comercio. 
FIGURA 2

COMARCALIZACIÓN COMERCIAL DE LA COMUNIDAD VALENCIANA, 2012, GRANDES SUPERFICIES COMERCIALES Y PAC

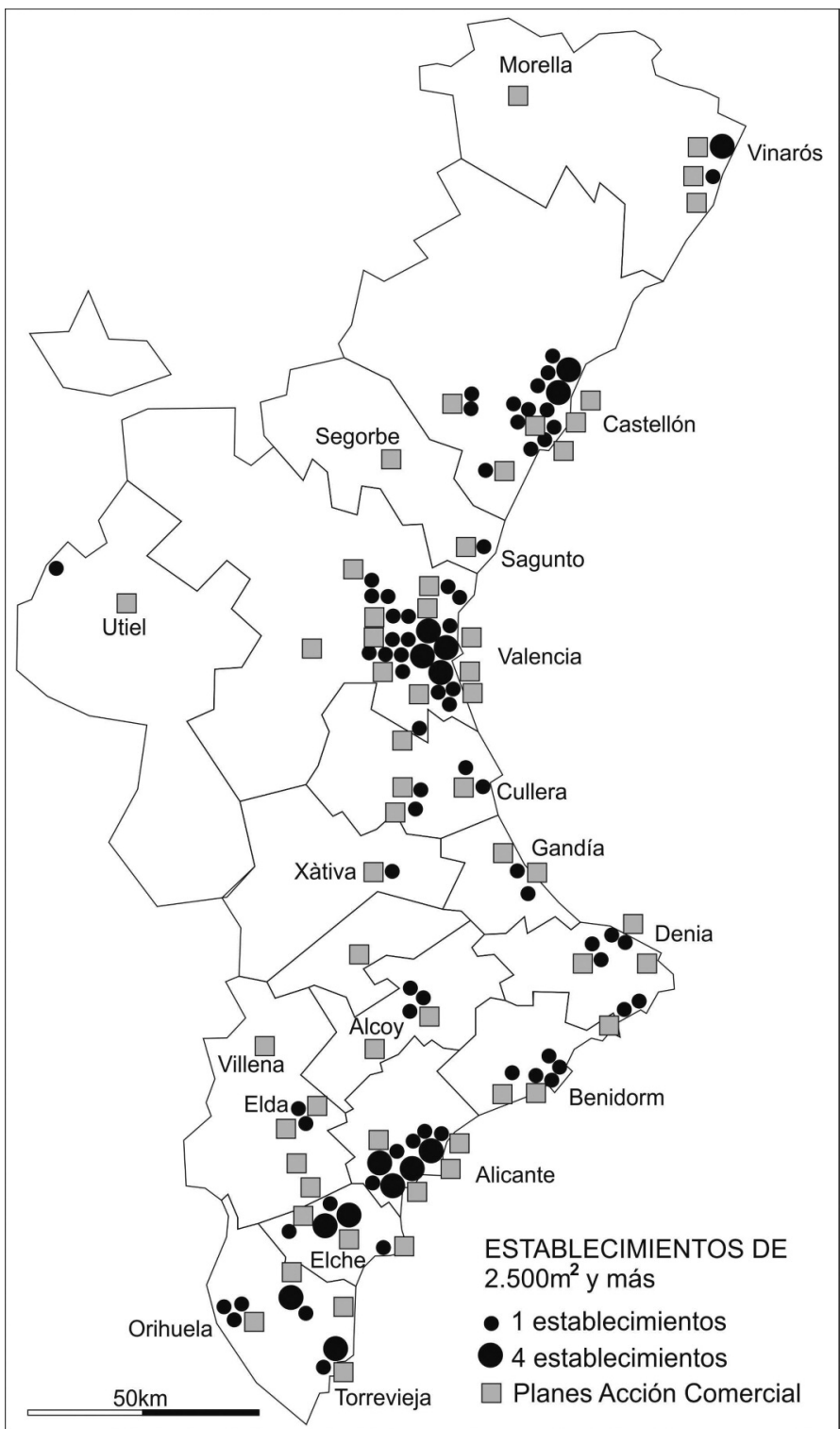

Fuente: Oficina Comercio y Territorio, 2013.

Estudios Geográficos, Vol. LXXV, 276, pp. 347-372, enero-junio 2014

ISSN: 0014-1496, eISSN: 1988-8546, doi: 10.3989/estgeogr.201409 
Los trabajos de realización de un PAC, de carácter bienal, plantean un enfoque transversal que asocia los diagnósticos sociodemográficos, económico-comerciales y urbano-comerciales para trazar las propuestas de actuación: ejes comerciales, centros de atracción, polos de nueva centralidad, zonas especializadas y áreas deficitarias de actividad comercial. Los planes de actuación, estimados para un horizonte de entre 5 y 7 años, se han centrado en propuestas de modernización de los equipamientos comerciales (mercados, mercadillos, establecimientos tradicionales), en actuaciones de urbanismo comercial (peatonalización, mobiliario urbano, paisajismo, señalética, accesibilidad y aparcamientos), y en propuestas de mejora de la competitividad empresarial (asociacionismo, cooperativismo, gestión conjunta de algunos servicios, capacitación y nuevas técnicas). Sin aportar datos concretos, la Oficina de Comercio y Territorio señalaba, en 2010, que los municipios que contaban con un PAC recibían mas subvenciones y ayudas, en las convocatorias públicas de la Generalitat Valenciana, para el desarrollo de programas sectoriales y de urbanismo comercial, que los que no contaban con ese plan (Oficina, 2010), lo que sin duda ha supuesto un acicate añadido para los intereses municipales. En el periodo 1998-2008, con 38 PAC, se había actuado en 468 calles, generando una inversión aproximada de 195 millones de euros (45 millones de inversión pública y otros 150 millones de inversión privada inducida) (Oficina, 2010).

Sin criterios territoriales previos, los planes se han desarrollado en cualquier tipo de municipio, siempre a instancias de los ayuntamientos interesados, sin que del mapa dibujado (figura 2) se puedan extraer más conclusiones que las siguientes:

- Son ciudades integradas en las principales aglomeraciones urbanas, con importantes áreas de atracción comercial suburbana y periurbana

- Son ciudades de la franja litoral, que aprovechan el PAC para regenerar sus centros tradicionales, como estrategia complementaria del turismo

- Son ciudades vecinas, afectadas por los grandes equipamientos comerciales suburbanos, que reaccionan a la feroz competencia

- Son municipios con grandes superficies comerciales, o afectados por las de municipios vecinos

- Menos frecuente son las ciudades industriales del interior, también afectadas por la instalación de grandes superficies comerciales suburbanas, que intentan regenerar sus espacios centrales de comercio

- Más raros son los municipios rurales de interior, alejados del turismo de masas, que han aprovechado los PAC para reforzar su atractivo como cabeceras comarcales de servicios. 
Pese a ello, en 2010 la Oficina de Comercio y Territorio, a efectos clasificatorios, agrupaba los PAC según el tamaño de los municipios en grandes (las capitales provinciales y Elche), medianos (entre 10.000 y 75.000 habitantes) y pequeños (menos de 10.000 habitantes). Es el PATSECOVA, presentado en 2012, el que propone medidas de equilibrio territorial para el futuro, entre los municipios y las áreas comerciales valencianas.

\subsection{Los resultados de los Planes de Acción Comercial}

Señalada la contradicción de comenzar desde la escala local, debe indicarse que los PAC han desarrollado una importante misión para contrarrestar en cierta medida las potentes tendencias suburbanizadoras del comercio minorista. En 2013, las 52.714 empresas de comercio al por menor radicadas en la Comunidad Valenciana (con 65.168 locales) suponían el 15,6\% del total de las empresas valencianas, superando ampliamente el número de empresas de otros importantes ramos de actividad, como la industria manufacturera (21.881 empresas) y las dedicadas a la construcción (43.872 empresas). Es pues actividad muy destacada en la Comunidad Valenciana, que ocupaba al $10,5 \%$ del total de la población empleada en ese año, generaba el 11,3\% del PIB (cuando la media de España era del 9,6\%), si bien, tan sólo contribuía con

\section{TABLA 1}

ESTRUCTURA ECONÓMICA. DATOS BÁSICOS EN LA EVOLUCIÓN DEL COMERCIO AL POR MENOR EN LA COMUNIDAD VALENCIANA Y ESPAÑA

\begin{tabular}{lrrrrrrr}
\hline & año 1999 & \multicolumn{2}{c}{$\%$ sobre total actividad } & & \multicolumn{2}{c}{ \% sobre total actividad } \\
\cline { 3 - 4 } & & C. Valenciana & España & & C. Valenciana & España \\
\hline empresas & 57.559 & 21,6 & 21,0 & 52.714 & 15,6 & 15,2 \\
locales & 68.786 & 23,1 & 21,2 & 65.168 & 17,1 & 16,7 \\
empleados & 151.345 & 9,1 & 8,8 & 182.927 & 10,5 & 9,5 \\
sueldos* & 1.200 .565 & 4,0 & 5,4 & 2.104 .406 & 4,8 & 4,2 \\
sin asalariados & 40.521 & 58,9 & 57,5 & 34.089 & 52,3 & 50,6 \\
*miles $€$ & & & & & & \\
\hline
\end{tabular}

Fuente: INE, elaboración propia. 
el $4,8 \%$ del total de sueldos y salarios, conforme con la muy marcada estructura familiar de las empresas, donde el 52\% (un total de 27.967 empresas en ese año) no contaba con ningún asalariado. Conviene pues precisar su ponderado papel como ramo generador de empleo, ya que en buena medida (siempre más de la mitad en la serie analizada: 1999-2012) se trata de actividad centrada en el autoempleo bajo la figura de autónomo.

A la vista de los grandes datos económicos del ramo y su evolución comparada con el conjunto de España (tabla 1), parece evidente que las estrategias de los PAC no han conseguido mitigar los efectos de la crisis, ni marcar una tendencia diferente a la experimentada en el conjunto del Estado. De hecho, la reducción del pequeño comercio minorista tradicional, el amparado en los PAC, va ligada al aumento del índice de superficie comercial por formatos, como ponen de manifiesto el aumento de las medianas y grandes superficies especializadas y mixtas (tabla 2).

TABLA 2

EVOLUCIÓN DEL ÍNDICE DE SUPERFICIE COMERCIAL DE LA COMUNIDAD VALENCIANA POR RAMOS Y POR FORMATOS

\begin{tabular}{lrrrr}
\hline Formato & 1991 & 1996 & 2005 & 2010 \\
\hline Productos de alimentación, bebidas y perfumería & 197 & 187 & 201 & 191 \\
Equipamiento personal & 148 & 154 & 151 & 166 \\
Equipamiento del hogar & 260 & 269 & 413 & 249 \\
Otro comercio al por menor & 118 & 129 & 262 & 198 \\
Medianas superficies especializadas & - & - & 157 & 117 \\
Grandes superficies especializadas (+2.500 m2) & - & 22 & 76 & 65 \\
Comercio mixto al por menor, por secciones & 223 & 277 & 398 & 374 \\
Autoservicios (40-120 m2) & 34 & 35 & 30 & 14 \\
Superservicios(121-399 m2) & 25 & 40 & 41 & 18 \\
Supermercados (400-2.500 m2) & 92 & 93 & 187 & 185 \\
Grandes superficies de alimentación (+2.500 m2) & 30 & 73 & 81 & 72 \\
Otros (discount, conveniencia, etc) & - & 6 & 35 & 43 \\
Grandes almacenes & 34 & 30 & 35 & 43 \\
\hline
\end{tabular}

Fuente: PATSECOVA, 2012, elaboración propia. 
Con todo, en el periodo de desarrollo de los PAC se observan claramente dos etapas definidas por los efectos de la crisis en 2008 (figura 3 y tabla 3). En la primera, las cifras generales son ligeramente ascendente para todos los estratos de asalariados contemplados, salvo para las empresas sin asalariados, que descienden. Puede entenderse que la bonanza económica, y tal vez los efectos positivos de los PAC, permitieron la contratación de algunos asalariados incluso en las empresas más pequeñas, que evolucionaron hacia otros estratos con más asalariados. Sin embargo, desde 2008 todos los estratos de empresas minoristas descienden, conforme con el retroceso general del número de empresas comerciales.

FIGURA 3

EVOLUCIÓN DEL NÚMERO DE EMPRESAS COMERCIO AL POR MENOR EN LA COMUNIDAD VALENCIANA, SEGÚN ESTRATOS DE ASALARIADOS

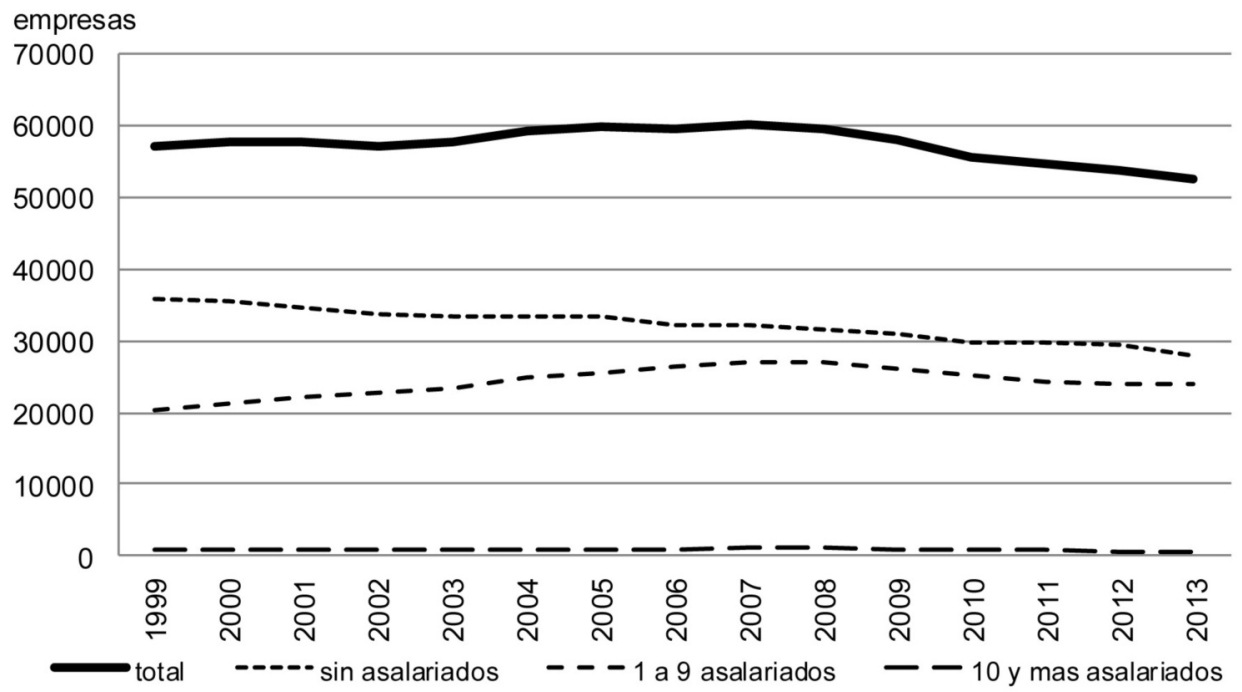

Fuente: INE, 2013, elaboración propia. 
TABLA 3

EVOLUCIÓN DEL NÚMERO DE LOCALES, OCUPADOS Y CIFRA NEGOCIO

(MILLONES €) EN EL COMERCIO AL POR MENOR DE LA COMUNIDAD VALENCIANA

\begin{tabular}{cccc}
\hline año & locales & personal ocupado & negocio (millones $€$ ) \\
\hline 1999 & 64.481 & 151.345 & 13.630 \\
2000 & 64.525 & 160.443 & 14.867 \\
2001 & 65.130 & 157.844 & 15.911 \\
2002 & 68.709 & 178.346 & 17.742 \\
2003 & 67.371 & 180.893 & 19.052 \\
2004 & 68.855 & 188.127 & 19.899 \\
2005 & 69.461 & 195.153 & 21.668 \\
2006 & 69.879 & 205.014 & 22.015 \\
2007 & 71.297 & 210.331 & 23.688 \\
2008 & 69.268 & 210.860 & 25.909 \\
2009 & 70.024 & 196.781 & 23.451 \\
2010 & 65.230 & 189.674 & 23.699 \\
2011 & 62.851 & 185.843 & 23.121 \\
2012 & 63.034 & 182.927 & 22.101 \\
\hline
\end{tabular}

Fuente: INE, elaboración propia.

En el periodo de estudio, la evolución de los puntos de venta en el comercio minorista en los municipios con PAC pone de manifiesto una considerable disparidad en la eficacia de las propuestas. De las 56 ciudades con PAC, la evolución del número de comercios minoristas ha sido favorable tan sólo en 13 de ellas, mientras las demás han experimentado en diverso grado una pérdida sustancial de ese equipamiento. Salvo en Torrevieja, la tasa de variación positiva ha sido muy moderada, siempre por debajo del 10\%, y su distribución positiva, junto con las pérdidas más moderadas, se ha dado entre los municipios de alta especialización turística (figura 4). Por el contrario, las mayores pérdidas parecen repartirse por las ciudades del interior y, también en el entorno metropolitano de Valencia, probablemente afectadas por la expansión de las grandes superficies. 
FIGURA 4

EVOLUCIÓN DE LOS PUNTOS DE VENTA EN LOS MUNICIPIOS CON PAC, PERIODO 1999-2012

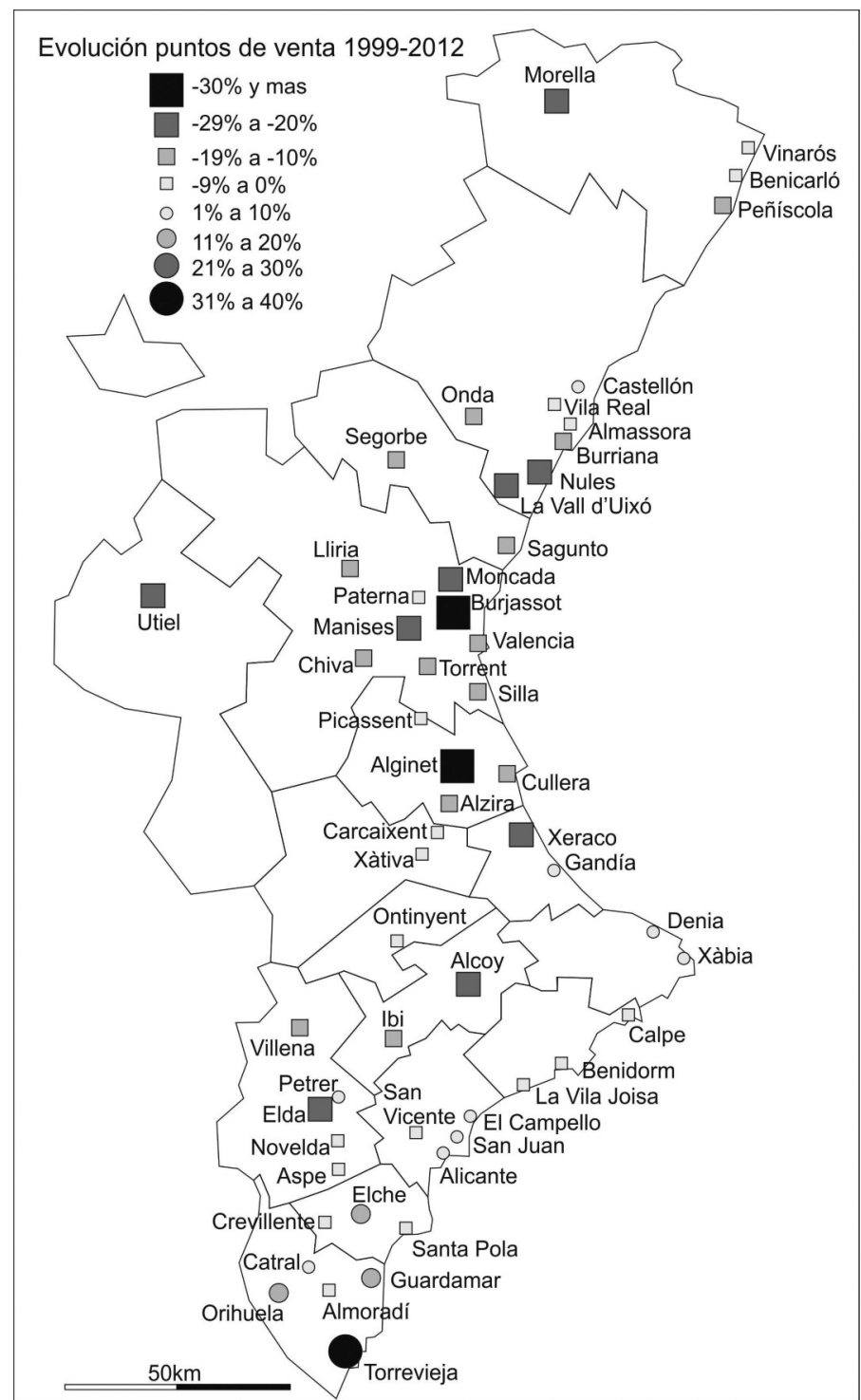

Fuente: Anuario Económico de España (La Caixa), y Oficina de Comercio y Territorio 2012, elaboración propia.

Estudios Geográficos, Vol. LXXV, 276, pp. 347-372, enero-junio 2014

ISSN: 0014-1496, eISSN: 1988-8546, doi: 10.3989/estgeogr.201409 
De hecho, en el periodo, la superficie de centros comerciales regitrada en 25 de esos municipios con PAC ascendió desde los $463.194 \mathrm{~m}^{2}$ hasta el $1.385 .270 \mathrm{~m}^{2}$ (Caixa, 2013), que suponen el $78 \%$ del total de la superficie de centros comerciales registrada en el conjunto de la Comunidad Valenciana.

\section{UN VERDADERO Y DEMORADO PAT SECTORIAL: EL PATSECOVA}

El PAT, promovido por la Dirección General de Comercio y Consumo de la Consellería de Economía y centrado en el comercio minorista, retoma los viejos objetivos de promover una estructura comercial equilibrada y competitiva, definir un modelo de planificación territorial sostenible del comercio y recuperar los ámbitos comerciales degradados (Cámaras, 2012). Su objetivo esencial es el de establecer un marco coherente, «de obligada referencia», para las Administraciones locales, para que el planeamiento urbanístico local contemple la actividad como agente importante en la construcción física y social de la ciudad y, a partir de ese criterio, proceder a autorizar o no los usos y los equipamientos comerciales, así como los planes y programas sectoriales (urbanismo comercial) pertinentes. Para ello, se explicita que en la documentación del PATSECOVA se incorporará una parte vinculante y dispositiva de obligada incorporación en los planes generales.

No obstante, se insiste en que el plan «no tomará en consideración el impacto de la implantación de nuevos establecimientos comerciales sobre la oferta comercial existente». Es decir, no servirá para atender una de las principales demandas de los comerciantes minoristas, y las propuestas y estrategias que trace para ese sector (y para los ámbitos urbanos interesados), pueden quedar desvirtuadas por la instalación de nuevos establecimientos, en el municipio o en otros colindantes.

\subsection{El nuevo mapa del comercio minorista}

Para intentar corregir los grandes desequilibrios existentes en el equipamiento comercial, el PATSECOVA elabora un nuevo mapa (figura 5) ahora si apoyado en una estructura polarizada y jerarquizada, si bien se indica «la imposibilidad de corregir efectos no deseables de acumulación de tipologías o formatos comerciales» (Cámaras: 2012: 109). Atendiendo a la realidad geográfica y socioeconómica de la Comunidad Valenciana, se identifican tres grandes zonas: la Cota cero (litoral por debajo de los $100 \mathrm{~m}$ de altitud) que re- 
FIGURA 5

DIRECTRICES TERRITORIALES BÁSICAS PARA LA DISTRIBUCIÓN DE LA ACTIVIDAD COMERCIAL

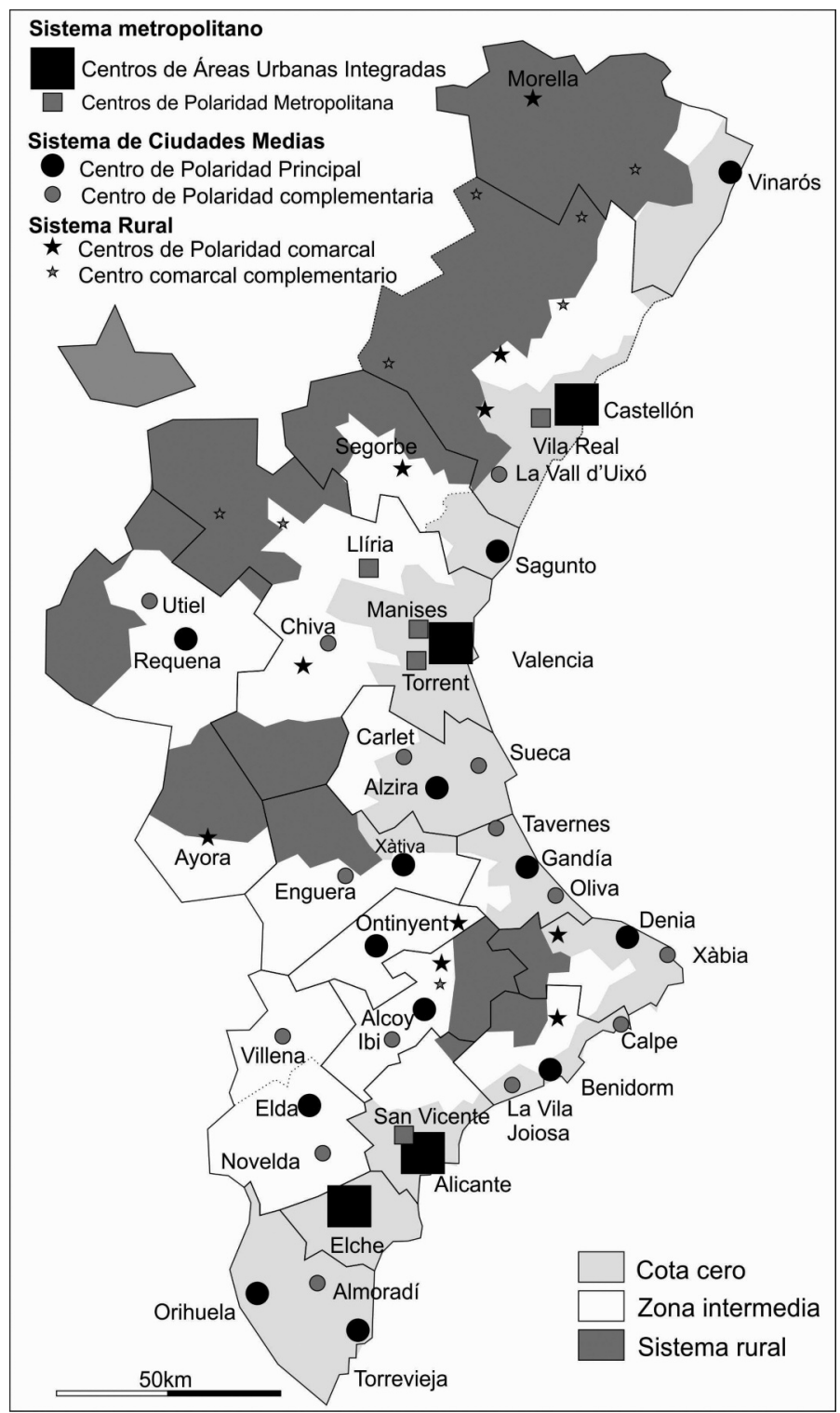

Fuente: Cámaras, 2012, elaboración propia.

Estudios Geográficos, Vol. LXXV, 276, pp. 347-372, enero-junio 2014

ISSN: 0014-1496, eISSN: 1988-8546, doi: 10.3989/estgeogr.201409 
úne el $80 \%$ de la población y al 96\% de las actividades comerciales minoristas, la Zona Intermedia, con el 17\% de la población, y el Sistema Rural, con el 3\% de la población. Sobre esa base, a partir del análisis de las zonas de atracción comercial de las diferentes ciudades, se llega a la delimitación de comarcas funcionales comerciales y a ponderar el peso gravitacional de los principales nodos de atracción. Se llega así a definir un Sistema Metropolitano, una Sistema de Ciudades Medias y un Sistema Rural prácticamente igual al definido por el Avance del Plan de Desarrollo Urbanístico de la Comunidad Valenciana (COPUT, 1995), que sirvió de base para vertebrar el territorio valenciano con dotaciones y equipamientos públicos, pero que fue arrinconado para la planificación territorial sectorial. En fase de Avance, su eficacia se verá condicionada por el desarrollo de los PAC locales (los vigentes y los que precisan una revisión) y sobre todo por el grado de seguimiento que se de en los planes generales y por las alteraciones resultantes de las actuaciones consideradas estratégicas o aprobadas con criterios de interés social.

\subsection{El papel del comercio en la ordenación territorial y urbanística de la Comunidad Valenciana}

La Ley Urbanística Valenciana (Ley 16/2005) ya recogía en sus postulados y articulado algunas reglamentaciones que vinculan la actividad comercial con la ordenación territorial y urbana. Entre otros más genéricos, se señalaba la necesaria inclusión dentro de la red primaria de reservas de suelo todos los equipamientos privados que contribuyan a la articulación de la ciudad, los susceptibles de generar tránsito intenso, los usos permitidos en cada inmueble -entre ellos el comercial-, las reservas para uso terciario en los sectores de uso residencial y las dotaciones de aparcamientos ( 1 plaza por cada $25 \mathrm{~m}^{2}$ construidos de zona comercial).

El PATSECOVA contempla también las novedades que introduce la ley estatal de Ordenación del Comercio Minorista (2010), en la que se consagra la libertad de establecimiento como principio general, eliminando la obligatoriedad de la licencia comercial (aunque puedan establecerse algunas regulaciones). Donde se suprime la definición de gran establecimiento comercial «para evitar el trato discriminatorio» que padecían en las autorizaciones. Aunque se considera factor limitante los posibles impactos en el medio ambiente, en el entorno urbano y en la conservación del patrimonio histórico, y se señala de manera explícita que los planes generales deben apoyar el comercio de proximidad -de barrio- como el más sostenible. 
En ese sentido, la Ley del Comercio de la Comunidad Valenciana (2011) apuntaba que la implantación quedará sujeta al impacto de los nuevos establecimientos comerciales sobre la oferta existente, incluido el impacto supramunicipal. La Ley considera que todo establecimiento de $2.500 \mathrm{~m}^{2}$ o más superficie es susceptible de provocará impacto y, por ello, su implantación deberá ser reglamentada. Entre otros aspectos, será tenida en cuenta la adecuación del establecimiento a las normas del PATSECOVA, su proporcionalidad respecto del entorno, su integración en el tejido urbano circundante, la suficiencia de la red de comunicaciones, las dotaciones de aparcamiento y de infraestructuras y servicios públicos y su articulación con la red de transporte público. Por todo ello, se manifiesta la necesidad de conciliar planificación sectorial comercial con ordenación urbanística, indicándose que los planes generales deberán calificar suelo comercial de manera diferenciada del conjunto de suelo terciario, incluyendo una clasificación por formatos.

\section{CONCLUSIONES}

El vaciamiento y deterioro de los espacios centrales de las ciudades valencianas (en sus aspectos poblacional y funcional) fue detonante de la voluntad de regular el proceso de implantación territorial de la actividad comercial, en sus diferentes formatos. Hecho que se manifestó tempranamente entre las tareas prioritarias del Gobierno Valenciano. Los Planes de Acción Territorial ofrecían la herramienta legal oportuna en los primeros años de autogobierno. Para elaborar el dedicado al comercio, se realizó un primer diagnóstico-Mapa del comercio de la Comunidad Valenciana (inédito)-, por convenio entre el Gobierno Valenciano y el Consejo de las Cámaras de Comercio de la Comunidad Valenciana. El cambio de gobierno regional y de modelos de gestión, amparados en el neoliberalismo y la postmodernidad conceptual, limitaron a partir de 1995 el desarrollo de los instrumentos de regulación urbanística de una actividad esencial para el urbanismo y la construcción de ciudad como espacio social. No obstante, sin una verdadera regulación territorial, se pusieron en marcha políticas de carácter marcadamente local -los PAC- cuyo objetivo ha sido el de apoyar al pequeño comercio minorista (de proximidad y de centro urbano) en su desigual batalla contra las medianas y grandes superficies suburbanas, de gran atracción e influencia periurbanas.

Del análisis de los diagnósticos y propuestas urbanísticas recogidas en los PAC, se desprende que los efectos del comercio sobre el urbanismo, con ser muy destacados, han sido siempre soslayados tanto en las tareas de planifica- 
ción territorial como en los planes generales locales. La implantación de los nuevos espacios comerciales se ha guiado básicamente por el interés de la empresa privada. En el ámbito sectorial, pese a las regulaciones, las grandes empresas han impuesto sus intereses sobre las pequeñas. En el ámbito territorial, la implantación de establecimientos comerciales, que ha sido tildada de espontánea y carente de planificación (Cámaras, 2012), ha seguido por el contrario una clara lógica, plasmada en precisas estrategias de búsqueda de la máxima accesibilidad para alcanzar la máxima cuota de mercado. Otra cuestión es que, en efecto, no haya existido una adecuada ordenación territorial, ni urbana -ni PAT, ni PG- para regular, no sólo la implantación, sino todos los efectos urbanísticos que se derivan de tan importante motor socioeconómico.

Analizadas las modestas propuestas urbanísticas de los PAC -urbanismo comercial- se comprueba como los planes generales no han valorado en suficiente medida el impacto del comercio. En general, es tratado como actividad envuelta en la calificación genérica de «Suelo Terciario», con pocas o ninguna regulación específica sobre el gran impacto urbanístico que ocasiona. Como norma, se ha considerado tan sólo como elemento complementario de los desarrollos residenciales o industriales, cuando en la práctica se ha manifestado en muchos casos, sobre todo en la periferia, como el motor y la clave de los mismos. Por lo común, los planes generales no han previsto ámbitos para el desarrollo de nuevos ejes comerciales y para el fomento del comercio de proximidad con continuidad en la trama urbana compacta. Al contrario, han delegado la planificación de esa actividad a los intereses privados, que por lo común, encuentra muchas más facilidades en las periferias que en el suelo urbano compacto. Puede concluirse pues que, además de las tendencias centrífugas del comercio recogidas como contexto teórico general, la falta de concisión del comercio como actividad fundamental en los planes generales valencianos ha fomentado su éxodo hacia las periferias.

La deslocalización industrial, que afecta a la Comunidad Valenciana desde los años 1980, ha vaciado de actividad grandes polígonos industriales, que han sido paulatinamente colonizados por los grandes formatos comerciales, sobre todo los espacios más accesibles (en proceso de colonización comercial que avanza desde las fachadas de los polígonos hacia el interior de los mismos). El proceso ha transformado drásticamente la ocupación del suelo, forzando, o soslayando, las determinaciones de usos (superficie, intensidad, localización) recogidas en las normas de regulación de cada sector urbanístico. Se ha permitido el desarrollo comercial en tanto actividad terciaria compatible con el uso determinado por el plan para el sector (uso industrial), pero alterado el uso, han aparecido nuevos flujos y dinámicas espaciales no previs- 
tas en los antiguos planes, con notable incidencia en los propios polígonos -equipamientos e infraestructuras-y en los ámbitos urbanos adyacentes, afectados además por nuevos procesos especulativos que contribuyen a alterar también las previsiones anteriores para el uso de cada tipo de suelo.

Los mismos PAC son un remedo de la falta de atención a la actividad comercial en los centros urbanos. En general, las normas urbanísticas que regulan los centros históricos, e incluso los ensanches, introducen considerables limitaciones a la actividad comercial, conforme con la evolución experimentada por el sector. Los condicionantes derivados del entramado urbano, de las tipologías edificatorias, de la protección del patrimonio se han sumado a claros limitantes en el aspecto logístico de esos ámbitos (almacenamiento, reparto, superficies comerciales de los establecimientos, aparcamientos). Todo ello sumado a los procesos ya expuestos de envejecimiento y despoblación de los centros urbanos.

Frente a tales dinámicas, los resultados generales de la evolución del comercio minorista en la etapa de vigencia de los PAC 1999-2012, ponen de manifiesto la insuficiencia de esos planes locales (de hecho planes de barrio: el barrio central) para la supervivencia del sector minorista: en su conjunto -como evidencia la pérdida general de establecimientos en 43 de los 56 municipios con PAC- y en concreto en los espacios del comercio tradicional, afectados por el crecimiento constante de los centros comerciales -un $200 \%$ en el conjunto de los municipios con PAC en el periodo analizado-.

Con todo, los diagnósticos y propuestas de los PAC han conseguido concienciar al tejido empresarial tradicional que, en algunos casos, siempre de manera modesta, han iniciado programas de renovación sectorial y cohesión empresarial para algunas estrategias de cooperación, en línea con el concepto de centro comercial abierto, aunque el proceso es muy incipiente y se ha visto muy afectado por la crisis económica desde el año 2008.

Desde el punto de vista territorial, puede concluirse que la pugna sectorial entre pequeños y grandes comerciantes, antes librada en el interior de las ciudades valencianas, ahora se ha trasladado a un conflicto entre centro -decadente- y periferias -pujantes-, que ha traspasado el ámbito meramente empresarial, afectando a la propia construcción urbana y al modelo social. En ese sentido, destacado es el nuevo papel de los operadores privados en la construcción de nueva ciudad a partir de la calificación (en el sentido especulativo) del suelo por la implantación de nuevos centros comerciales, diseñados de hecho como nuevas centralidades sociales.

Es el PATSECOVA el que propone, en su Avance de 2012, la regulación territorial de esas dinámicas, señalando la conveniencia de incluir determina- 
ciones específicas, de obligada observación en los planes generales, y directrices de coordinación territorial, en busca de equilibrar la oferta comercial, entre centros y periferias y entre grandes polos de atracción saturados -áreas metropolitanas- y el resto del territorio valenciano. Es documento esencial, que debe ser aprobado y consensuado para configurar un futuro mapa del comercio minorista, en el que prevalezca su función territorial en la construcción de ciudad y de modelos sociales, y luego respetado por los planes generales locales y, también, por las potenciales (y esperadas) Actuaciones Territoriales Estratégicas (ATE: Decreto-Ley 2/2011) «para generar empleo» que auspicia la Estrategia Territorial de la Comunidad Valenciana (Gobierno Valenciano, 2011), en la que se contempla como ejemplo de ATE los «nodos singulares de actividad comercial, para acoger actividades comerciales avaladas por una marca de prestigio internacional y de ratio de influencia provincial como mínimo, o bien por una concentración comercial singular y diferenciada que genere masa crítica suficiente para desarrollar una oferta cuya área de captación supere el ámbito comarcal de los grandes centros comerciales al uso».

Fecha de recepción: 04/06/2013.

Fecha de aceptación 20/02/ 2014.

\section{Bibliografía Y FUENTES}

Blázquez, A. M. (2007): "Instrumentos de ordenación y gestión territorial y medioambiental. Marco normativo regulador de las actividades económicas en la Comunidad Valenciana". Cuadernos de Geografía, 81-82, pp. 133-160.

Boira, J. V. (2012): Valencia, la tormenta perfecta. Barcelona, RBA, 221 pp.

Boira (2013): "Vigencia del plan general, actividad inmobiliaria y modelo de ciudad en Valencia (1979-2010)", en: Metamorfosis urbanas. Barcelona, Icaria editorial, pp. 133-156.

Burriel, E. (2008): "La 'década prodigiosa' del urbanismo español (1997-2006)". Scripta Nova. Revista Electrónica de Geografía y Ciencias Sociales, XII/270 (64), http://www.ur.es/geogrit/sn/sn-270/sn-270-64.htm (Fecha de consulta: 22/05/2014).

Burriel, E. (2009a): "Los límites del planeamiento urbanístico municipal, el ejemplo valenciano". Documents d'Anàlisi Geogràfica, 54, pp. 35-54.

Burriel, E. (2009b): "La planificación territorial en la Comunidad Valenciana (19852009)". Scripta Nova. Revista Electrónica de Geografía y Ciencias Sociales, XIII (306), http://www.ur.es/geogrit/sn/sn-306.htm (Fecha de consulta: 22/05/2014).

Burriel, E. (2009c): "La Unión Europea y el urbanismo valenciano". Boletín de la Asociación de Geógrafos, 49, pp. 5-24. 
Caixa (2013): Anuario Económico de España. www.anuarieco.lacaixa.comunicacions. com (Fecha de consulta: 10/01/2014).

Consejo Cámaras Comunidad Valenciana (2009): Atlas Sociocomercial de la Comunidad Valenciana. Valencia, Oficina Comercio y Territorio del Consejo de Cámaras de la Comunidad Valenciana, 831 pp.

Consejo Cámaras Comunidad Valenciana, Conselleria d'Economia, Indústria, Turisme i Ocupació (2012): Plan de Acción Territorial del Comercio de la Comunidad Valenciana, PATSECOVA. www.patsecova.es (Fecha de consulta: 20/12/2013).

Conselleria de Territori i Habitatge (2006): Plan de Acción Territorial del Litoral. http://www.upv.es/contenidos/CAMUNISO/info/U0556814.pdf (Fecha de consulta: 6/04/2013).

Consellería d'Obres Públiques, Urbanismo i Transports (1995): Plan de desarrollo urbanístico de la Comunidad Valenciana, Síntesis del avance. Valencia, COPUT, 99 pp.

Comisión Europea (1995): Europa 2000+ Cooperación para la ordenación del territorio europeo. Políticas regionales de la Comunidad Europea. Luxemburgo, Comisión Europea, $247 \mathrm{pp}$.

Cucó, J. (ed.) (3013): Metamorfosis urbanas. Barcelona, Icaria editorial, 399 pp.

Cucó, J. (2013): "Poniendo a Valencia en el mapa global. Políticas, desarrollos urbanos y narrativas sobre la ciudad", en J. Cucó (ed.): Metamorfosis urbanas. Barcelona, Icaria editorial, pp. 157-180.

Del Romero, L. (2010): "Dos décadas deurbanismo-espectáculo en España: los grandes eventos como motor de cambio urbano". Boletín de la AGE, 53, pp. 309-327.

Díaz Orueta, F. (2013): "Sociedad, espacio y crisis en la ciudad neoliberal”, en J. Cucó (ed.): Metamorfosis urbanas, Barcelona, Icaria editorial, pp. 157-180.

Feria, J. M. (2010): "La movilidad residencial y los procesos de urbanización metropolitanos en España”, en Juan Miguel Albertos Puebla y José María Feria Toribio: La ciudad metropolitana en España: procesos urbanos en los inicios del siglo XXI. Pamplona, CIVITAS, pp. 23-47.

Gobierno Valenciano (2011): Estrategia Territorial de la Comunidad Valenciana. http://cma.gva.es/web/indice.aspx nodo=58253\&idioma $=C$ (Fecha de consulta: 5/01/2013).

Hermosilla, J. (dir.) (2000): Las grandes superficies comerciales del área Metropolitana de Valencia. Valencia, Universidad de Valencia, $187 \mathrm{pp}$.

Ministerio de Fomento (2013): Atlas Estadístico de las Áreas Urbanas de España. https://www.fomento.gob.es (Fecha de consulta: 8/09/2014).

Hermosilla, J. y Rodrigo, C. (1997): Las grandes superficies comerciales en la Comunidad Valenciana. Influencia sobre el pequeño comercio. Ribarroja del Turia, Ayuntamiento Ribarroja, 160 pp.

Nel.lo, O. (1998): "Transformaciones y políticas urbanas en las siete principales ciudades de España. El área metropolitana de Valencia en el sistema urbano español”. Área Metropolitana. Revista informativa del CMH, 4, pp.4-9. 
Oficina Comercio y Territorio, (2010): Los Planes de Acción Comercial, 1999-2010. Valencia, Consejo de Cámaras de la Comunidad Valenciana, 21 pp.

Salom, J. (1992): Sistema urbano y desarrollo regional en la comunidad Valenciana. Valencia, Edicions Alfons el Magnànim-IVEI, 246 pp.

Ponce, G. (2005): "De la taylorización a la vertebración del territorio valenciano". Ciudad y Territorio, XXXVII/143, pp. 105-124.

Ponce, G. (2006): "La fragmentación de la forma urbana en la Comunidad Valenciana”, en G. J. Ponce Herrero: La ciudad fragmentada. Nuevas formas de hábitat. Alicante, Universidad de Alicante, pp. 89-130.

Ponce, G. y Martí, P. (2011): "De archipiélago urbano a bulevar litoral. La expansión urbana en el litoral mediterráneo", en: Urbanismo expansivo: de la utopía a la realidad. Alicante, AGE, pp. 541-554.

Roca, J.; Moix, M. y Arellano, B. (2012): "El sistema urbano en España". Scripta Nova, XVI/395. http://www.ub.es/geocrit/sn/sn-396.htm (Fecha de consulta: 10/06/2014).

Salom, J. y Albertos, J. M. (2010): "El modelo de desarrollo de la Comunidad Valenciana", en Juan Romero, Alfredo Morales, Julia Salom y Fernando Vera (coords.): La periferia emergente. La Comunidad Valenciana en la Europa de las regiones. Barcelona, Ariel, pp. 65-82.

Salom, J. y Albertos, J. M. (2010): "Densidad de la red viaria y forma urbana: delimitación del espacio urbano en ocho aglomeraciones españolas", en: La ciudad metropolitana en España: procesos urbanos en los inicios del siglo XXI. Navarra, Civitas, pp. 49-94.

\section{RESUMEN}

El modelo de ciudad expansiva, ampliamente desarrollado en la Comunidad Valenciana, ocasiona cambios de los hábitos ciudadanos y, con ellos, de sus pautas de consumo, mediatizadas por el atractivo diseño de los nuevos centros comerciales y, en especial, por su vinculación con el ocio. Tales procesos alteran sustancialmente el modelo comercial y de relación social de la ciudad. Los primeros gobiernos de la Comunidad Valenciana desarrollaron un aparato legal que pretendía regular y aprovechar el papel del comercio como herramienta de vertebración social y territorial. Fruto de ello son los Planes de Acción Comercial, para la salvaguarda y mantenimiento de los antiguos espacios comerciales de la ciudad (centros históricos y Ensanches), muy afectados por el cambio. Son programas económicos, con un modesto soporte urbanístico, para recuperar el atractivo económico perdido y regenerar la capacidad de la ciudad para el encuentro y la relación. Sin embargo, por su carácter puntual, no han logrado contrarrestar los grandes movimientos centrífugos del equipamiento comercial.

PAlabras Clave: autogobierno; estrategias regionales y territoriales; comercio minorista. 


\begin{abstract}
The expansive city model extensively developed by the Comunidad Valenciana, has led to changes in the habits and customs of its citizens which in turn has led to alterations in the way they consume, seduced by advertising and the attractive design of new shopping centres and, in particular their links with leisure activities. These processes have substantially modified the business model and the social relations in the city. The first governments of the Comunidad Valenciana developed legal apparatus designed to regulate and profit from the role of commerce as a tool for social and territorial cohesion. This resulted in Commercial Action Plans designed to safeguard and ensure the survival of older and more traditional commercial quarters of the city (historic centres and surroundings) which have been severely affected by these changes. They consist of financial programmes with a modest urban planning component designed to recover the lost financial incentives and regain the city's capacity for social interaction and relation. However, as it has tended to be a piecemeal effort, it has not been possible to counteract the major centrifugal movements of commercial facilities.
\end{abstract}

KEY WORDS: self government; regional and local strategies; retail trade.

\title{
RÉSUMÉ
}

Le modèle de ville expansive, qui s'est amplement développé dans la Communauté valencienne, provoque des changements dans les comportements des citadins et donc dans leurs habitudes de consommation, conditionnées par les designs très attractifs des nouveaux centres commerciaux et surtout par la vocation de ceux-ci à être des lieux de loisirs. Ces processus transforment profondément le modèle commercial et les modes de relation sociale dans les villes. Les premiers gouvernements autonomes de la Communauté valencienne ont mis en place un dispositif légal qui avait l'intention de réglementer et de profiter du rôle du commerce en tant qu'instrument de cohésion sociale et territoriale. Cela a donné lieu à des Plans d'Action Commerciale voués à la sauvegarde et au maintien des anciens espaces commerciaux urbains (centres historiques et zones d'expansion), très affectés par ces changements. Il s'agit de programmes économiques, dotés d'un support urbanistique modeste, destinés à récupérer l'attrait économique déchu et à régénérer la capacité des villes en tant que lieux de rencontre et de relation sociale. Cependant, dû à leur caractère ponctuel, ces plans ne sont pas parvenus à contrecarrer les grands mouvements centrifuges de l'équipement commercial.

MotS-CLÉS: gouvernement autonome; stratégies régionales et territoriales; commerce de détail. 